\title{
BRAID GROUPS IN COMPLEX PROJECTIVE SPACES
}

\author{
BARBU BERCEANU ${ }^{1,2}$, SAIMA PARVEEN ${ }^{2}$
}

\begin{abstract}
We describe the fundamental groups of ordered and unordered $k$-point sets in $\mathbb{C} P^{n}$ generating a projective subspace of dimension $i$. We apply these to study connectivity of more complicated configurations of points.
\end{abstract}

\section{INTRODUCTION}

Let $M$ be a manifold and $\mathcal{F}_{k}(M)$ be the ordered configuration space of $k$ distinct points $\left\{\left(x_{1}, \ldots, x_{k}\right) \in M^{k} \mid x_{i} \neq x_{j}, i \neq j\right\}$. There is a proper right action of $\Sigma_{k}$, the symmetric group of order $k$, on $\mathcal{F}_{k}(M)$. The orbit space $\mathcal{F}_{k}(M) / \Sigma_{k}$ is the unordered configuration space, denoted $\mathcal{C}_{k}(M)$, and the natural projection $\mathcal{F}_{k}(M) \longrightarrow \mathcal{C}_{k}(M)$ is a regular covering. For a simply connected manifold $M$ of dimension $\geq 3$, the pure braid group $\pi_{1}\left(\mathcal{F}_{k}(M)\right)$ is trivial and the braid group $\pi_{1}\left(\mathcal{C}_{k}(M)\right)$ is isomorphic to $\Sigma_{k}$; as an example, $\pi_{1}\left(\mathcal{F}_{k}\left(\mathbb{C} P^{k}\right)\right)=1$ for $k \geq 2$. In low dimensions there are non trivial pure braids. The pure braid group of the plane, denoted by $\mathcal{P} \mathcal{B}_{n}$, has the presentation $[\mathrm{F}$

$$
\pi_{1}\left(\mathcal{F}_{n}(\mathbb{C})\right)=\mathcal{P} \mathcal{B}_{n} \cong\left\langle\alpha_{i j}, 1 \leq i<j \leq n \mid(Y B 3)_{n},(Y B 4)_{n}\right\rangle
$$

where the Yang-Baxter relations $(Y B 3)_{n}$ and $(Y B 4)_{n}$ are, for any $1 \leq i<$ $j<k \leq n$,

$$
(Y B 3)_{n}: \alpha_{i j} \alpha_{i k} \alpha_{j k}=\alpha_{i k} \alpha_{j k} \alpha_{i j}=\alpha_{j k} \alpha_{i j} \alpha_{i k}
$$

and, for any $1 \leq i<j<k<l \leq n$,

$$
(Y B 4)_{n}:\left[\alpha_{k l}, \alpha_{i j}\right]=\left[\alpha_{i l}, \alpha_{j k}\right]=\left[\alpha_{j l}, \alpha_{j k}^{-1} \alpha_{i k} \alpha_{j k}\right]=\left[\alpha_{j l}, \alpha_{k l} \alpha_{i k} \alpha_{k l}^{-1}\right]=1 .
$$

The braid group of the plane, denoted by $\mathcal{B}_{n}$, has the classical Artin presentation [A]

$$
\pi_{1}\left(\mathcal{C}_{n}(\mathbb{C})\right)=\mathcal{B}_{n} \cong\left\langle\sigma_{i}, i=1, \ldots, n-1 \mid(A)_{n}\right\rangle
$$

where the Artin relations $(A)_{n}$ are

$$
(A)_{n}:\left\{\begin{array}{l}
\sigma_{i} \sigma_{j}=\sigma_{j} \sigma_{i}, \quad \forall i, j=1, \ldots, n-1 \text { with }|i-j| \geq 2 \\
\sigma_{i} \sigma_{i+1} \sigma_{i}=\sigma_{i+1} \sigma_{i} \sigma_{i+1} \text { for } i=1, \ldots, n-2 .
\end{array}\right.
$$

Key words and phrases: complex projective space, configuration spaces, braid groups, Pappus configuration

This research is partially supported by Higher Education Commission, Pakistan. 2010 AMS Classification Primary: 20F36, 52C35, 57M05; Secondary: 51A20. 
The pure braid and the braid groups of $S^{2} \approx \mathbb{C} P^{1}$ have the presentations B2,, $\mathrm{F}$

$$
\begin{gathered}
\pi_{1}\left(\mathcal{F}_{k+1}\left(S^{2}\right)\right) \cong\left\langle\alpha_{i j}, 1 \leq i<j \leq k \mid(Y B 3)_{k},(Y B 4)_{k}, D_{k}^{2}=1\right\rangle \\
\pi_{1}\left(\mathcal{C}_{k+1}\left(S^{2}\right)\right) \cong\left\langle\sigma_{i}, 1 \leq i \leq k \mid(A)_{k+1}, \sigma_{1} \sigma_{2} \ldots \sigma_{k}^{2} \ldots \sigma_{2} \sigma_{1}=1\right\rangle,
\end{gathered}
$$

where $D_{k}=\alpha_{12} \alpha_{13} \alpha_{23} \ldots \alpha_{1 k} \ldots \alpha_{k-1, k}$.

The inclusion morphisms $\mathcal{P} \mathcal{B}_{n} \rightarrow \mathcal{B}_{n}$ for $\mathbb{C}$ and $\mathbb{C} P^{1}$ are given by (see [B2])

$$
\alpha_{i j} \mapsto \sigma_{j-1} \sigma_{j-2} \ldots \sigma_{i+1} \sigma_{i}^{2} \sigma_{i+1}^{-1} \ldots \sigma_{j-1}^{-1}
$$

(due to these inclusions, we can identify the pure braid $D_{k}$ with $\Delta_{k}^{2}$, the square of Garside braid).

Using the geometrical structure of projective spaces we stratify the configuration spaces $\mathcal{F}_{k}\left(\mathbb{C} P^{n}\right)$ and $\mathcal{C}_{k}\left(\mathbb{C} P^{n}\right)$ with complex submanifolds as follows:

$$
\mathcal{F}_{k}\left(\mathbb{C} P^{n}\right)=\coprod_{i=1}^{n} \mathcal{F}_{k}^{i, n},
$$

where $\mathcal{F}_{k}^{i, n}$ is the ordered configuration space of all $k$ points in $\mathbb{C} P^{n}$ generating a subspace of dimension $i$, and

$$
\mathcal{C}_{k}\left(\mathbb{C} P^{n}\right)=\coprod_{i=1}^{n} \mathcal{C}_{k}^{i, n},
$$

where $\mathcal{C}_{k}^{i, n}$ is the unordered configuration space of all $k$ points in $\mathbb{C} P^{n}$ generating a subspace of dimension $i$. Obviously, $\mathcal{C}_{k}^{i, n} \cong \mathcal{F}_{k}^{i, n} / \Sigma_{k}$.

Theorem 1.1. The spaces $\mathcal{F}_{k}^{i, n}$ are simply connected with the following exceptions

(1) for $k \geq 2$,

$$
\pi_{1}\left(\mathcal{F}_{k+1}^{1,1}\right) \cong\left\langle\alpha_{i j}, \quad 1 \leq i<j \leq k \mid(Y B 3)_{k},(Y B 4)_{k}, D_{k}^{2}=1\right\rangle ;
$$

(2) for $k \geq 3$ and $n \geq 2$,

$$
\pi_{1}\left(\mathcal{F}_{k+1}^{1, n}\right) \cong\left\langle\alpha_{i j}, \quad 1 \leq i<j \leq k \mid(Y B 3)_{k},(Y B 4)_{k}, D_{k}=1\right\rangle .
$$

In this list of non simply connected spaces, only $\mathcal{F}_{3}^{1,1}$ has finite fundamental group and this is isomorphic to $\mathbb{Z}_{2}$.

Corollary 1.2. The first homology groups $H_{1}\left(\mathcal{F}_{k}^{i, n}\right)$ are trivial with the following exceptions

(1) for $k \geq 2, \quad H_{1}\left(\mathcal{F}_{k+1}^{1,1}\right)=\mathbb{Z}^{\left(\begin{array}{l}k \\ 2\end{array}\right)-1} \oplus \mathbb{Z}_{2}$;

(2) for $k \geq 3$ and $n \geq 2, \quad H_{1}\left(\mathcal{F}_{k+1}^{1, n}\right)=\mathbb{Z}^{\left(\begin{array}{c}k \\ 2\end{array}\right)-1}$.

Theorem 1.3. The fundamental group of $\mathcal{C}_{k}^{i, n}$ is isomorphic to $\Sigma_{k}$ with the following exceptions 
(1) for $k \geq 2$,

$$
\pi_{1}\left(\mathcal{C}_{k+1}^{1,1}\right) \cong\left\langle\sigma_{1} \ldots \sigma_{k} \mid(A)_{k}, \sigma_{1} \ldots \sigma_{k}^{2} \ldots \sigma_{1}=1\right\rangle ;
$$

(2) for $k \geq 3$ and $n \geq 2$,

$$
\pi_{1}\left(\mathcal{C}_{k+1}^{1, n}\right) \cong\left\langle\sigma_{1} \ldots \sigma_{k} \mid(A)_{k}, \sigma_{1} \ldots \sigma_{k}^{2} \ldots \sigma_{1}=1, \Delta_{k}^{2}=1\right\rangle,
$$

where $\Delta_{k}=\sigma_{1} \sigma_{2} \sigma_{1} \sigma_{3} \sigma_{2} \sigma_{1} \ldots \sigma_{k-1} \ldots \sigma_{1}$.

The space $\mathcal{C}_{k}^{i, n}$ has a finite fundamental group only for $2 \leq i \leq \min (k+$ $1, n)$, and in this case $\pi_{1}\left(\mathcal{C}_{k}^{i, n}\right) \cong \Sigma_{k}$, and also $\pi_{1}\left(\mathcal{C}_{3}^{1,1}\right)$ is the dicyclic group of order 12 .

Corollary 1.4. The homology groups $H_{1}\left(\mathcal{C}_{k+1}^{i, n}\right)$ are isomorphic to $\mathbb{Z}_{2}$ with the following exceptions

(1) for $k \geq 2, H_{1}\left(\mathcal{C}_{k+1}^{1,1}\right)=\mathbb{Z}_{2 k}$;

(2) for $k \geq 3$ and $n \geq 2, H_{1}\left(\mathcal{C}_{k+1}^{1, n}\right)=\left\{\begin{array}{l}\mathbb{Z}_{k} \text { for } k=\text { even, } \\ \mathbb{Z}_{2 k} \text { for } k=\text { odd } \text {. }\end{array}\right.$

V.L. Moulton studied in M2 a related problem: braids for $m$ points in general position in $\mathbb{C} P^{n}$ (any subset of $n+1$ points spans $\mathbb{C} P^{n}$ ) and the analogous affine problem. The only intersection of this paper with [M2] is the simply connectedness of $\mathcal{F}_{n+1}^{n, n}\left(Y_{n+1}^{n}\right.$ in Moulton notations).

Section 2 contains the geometrical part of the paper: local triviality of some natural fibrations associated to $\mathcal{F}_{k}^{i, n}$ and $\mathcal{C}_{k}^{i, n}$, and also in this section we meet the pure braid $\Delta_{k}^{2}\left(\Delta_{k}\right.$ is the fundamental Garside [G] braid in $\mathcal{B}_{k}$ ) playing the main role in homotopical computations (see Lemma 2.12).

The proofs of Theorems 1.1 and 1.3 and their Corollaries are given in Section 3.

In the last section we compute the fundamental groups of $\mathcal{P}$, Pappus' configuration space.

Theorem 1.5. The fundamental group of $\mathcal{P}$ is isomorphic to $\mathbb{F}_{2} \times \mathbb{F}_{2}$.

$\left(\mathbb{F}_{n}\right.$ is the free group with $n$ generators).

In the Appendix one can find some words representing $\Delta_{n}$ and $D_{n}=\Delta_{n}^{2}$.

\section{LOCAL TRIVIALITY OF GEOMETRIC FIBRATIONS}

We begin with some simple remarks on the stratification with complex submanifolds

$$
\mathcal{F}_{k}\left(\mathbb{C} P^{n}\right)=\coprod_{i=1}^{n} \mathcal{F}_{k}^{i, n} .
$$

Remark 2.1. (1) $\mathcal{F}_{k}^{i, n} \neq \emptyset$ if and only if $i \leq \min (k+1, n)$;

(2) $\mathcal{F}_{k}^{1,1}=\mathcal{F}_{k}\left(\mathbb{C} P^{1}\right), \mathcal{F}_{2}^{1, n}=\mathcal{F}_{2}\left(\mathbb{C} P^{n}\right)$;

(3) the adjacency of the strata is given by

$$
\overline{\mathcal{F}_{k}^{i, n}}=\mathcal{F}_{k}^{1, n} \coprod \ldots \coprod \mathcal{F}_{k}^{i, n} .
$$


Grasmannian manifolds are related to the spaces $\mathcal{F}_{k}^{i, n}$ through the following fibrations:

Proposition 2.2. The projection

$$
\gamma: \mathcal{F}_{k}^{i, n} \rightarrow G r^{i}\left(\mathbb{C} P^{n}\right)
$$

given by

$$
\left(x_{1}, \ldots, x_{k}\right) \mapsto i-t h \text { dimensional space generated by }\left\{x_{1}, x_{2}, \ldots, x_{k}\right\}
$$

is a locally trivial fibration with fiber $\mathcal{F}_{k}^{i, i}$.

Proof. Take $V_{0} \in G r^{i}\left(\mathbb{C} P^{n}\right)$ and choose $L_{0} \in G r^{n-i-1}\left(\mathbb{C} P^{n}\right)$ such that $L_{0} \cap V_{0}=\emptyset$ and let define $\mathcal{U}_{L_{0}}$, an open neighborhood of $V_{0}$, by

$$
\mathcal{U}_{L_{0}}=\left\{V \in G r^{i}\left(\mathbb{C} P^{n}\right) \mid L_{0} \cap V=\emptyset\right\} .
$$

Take $k$ distinct points $\left(x_{1}, \ldots, x_{k}\right)$ in $V_{0}$ such that $\operatorname{span}\left(x_{1}, \ldots, x_{k}\right)=V_{0}$. For any arbitrary $i$-plane $V$ in $\mathcal{U}_{L_{0}}$, define the projective isomorphism

$$
\varphi_{V}: V_{0} \rightarrow V, \varphi_{V}(x)=\left(x \vee L_{0}\right) \cap V .
$$

The local trivialization is given by the homeomorphism

$$
\begin{gathered}
f: \gamma^{-1}\left(\mathcal{U}_{L_{0}}\right) \rightarrow \mathcal{U}_{L_{0}} \times \mathcal{F}_{k}\left(V_{0}\right) \\
y=\left(y_{1}, \ldots, y_{k}\right) \mapsto\left(\gamma\left(y_{1}, \ldots, y_{k}\right),\left(\varphi_{\gamma(y)}^{-1}\left(y_{1}\right), \ldots, \varphi_{\gamma(y)}^{-1}\left(y_{k}\right)\right)\right)
\end{gathered}
$$

(where $\mathcal{F}_{k}\left(V_{0}\right) \approx \mathcal{F}_{k}^{i, i}$ ), making the diagram commutative

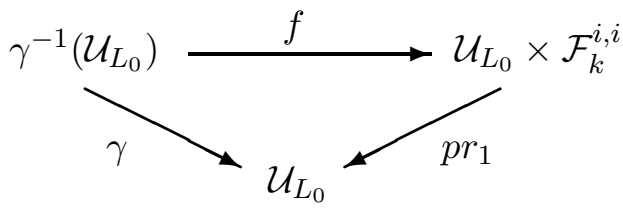

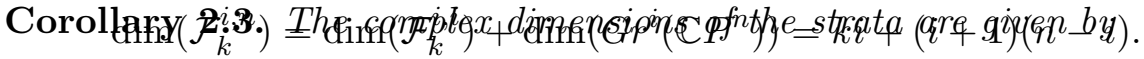

Proof. $\mathcal{F}_{k}^{i, i}$ is a Zariski open subset in $\left(\mathbb{C} P^{i}\right)^{k}$ for $k \geq i+1$.

Lemma 2.4. The projection

$$
p: \mathcal{F}_{k+1}^{k, k} \longrightarrow \mathcal{F}_{k}^{k-1, k}, \quad\left(x_{1}, \ldots, x_{k+1}\right) \mapsto\left(x_{1}, \ldots, x_{k}\right)
$$

is a locally trivial fibration with fiber $\mathbb{C} P^{k} \backslash \mathbb{C} P^{k-1} \approx \mathbb{C}^{k}$.

Proof. Take $\left(x_{1}^{0}, \ldots, x_{k+1}^{0}\right) \in \mathcal{F}_{k+1}^{k, k}$ and fix $x_{k+2}^{0}$ such that any $k+1$ points of the set $\left\{x_{1}^{0}, \ldots, x_{k+1}^{0}, x_{k+2}^{0}\right\}$ are independent. Define the open neighborhood $\mathcal{U}$ of $\left(x_{1}^{0}, \ldots, x_{k}^{0}\right)$ by

$$
\mathcal{U}=\left\{\left(x_{1}, \ldots, x_{k}\right) \mid \text { the set }\left\{x_{1}, \ldots, x_{k}, x_{k+1}^{0}, x_{k+2}^{0}\right\} \text { is in general position }\right\} .
$$


There exists a unique projective isomorphism $T_{\left(x_{1}, \ldots, x_{k}\right)}: \mathbb{C} P^{k} \longrightarrow \mathbb{C} P^{k}$, which depends continuously on $\left(x_{1}, \ldots, x_{k}\right)$, such that

$$
T_{\left(x_{1}, \ldots, x_{k}\right)}\left(x_{1}^{0}, \ldots, x_{k}^{0}, x_{k+1}^{0}, x_{k+2}^{0}\right)=\left(x_{1}, \ldots, x_{k}, x_{k+1}^{0}, x_{k+2}^{0}\right)
$$

(see [B1]). We can define the homeomorphisms $\varphi, \psi$ by :

$$
\begin{gathered}
p^{-1}(\mathcal{U}) \underset{\psi}{\stackrel{\varphi}{\rightleftarrows}} \mathcal{U} \times\left(\mathbb{C} P^{k} \backslash \operatorname{span}\left\{x_{1}^{0}, \ldots, X_{k}^{0}\right\}\right) \\
\varphi\left(x_{1}, \ldots, x_{k}, x\right)=\left(\left(x_{1}, \ldots, x_{k}\right), T_{\left(x_{1}, \ldots, x_{k}\right)}^{-1}(x)\right) \\
\psi\left(\left(x_{1}, \ldots, x_{k}\right), y\right)=\left(x_{1}, \ldots, x_{k}, T_{\left(x_{1}, \ldots, x_{k}\right)}(y)\right)
\end{gathered}
$$

satisfying $p r_{1} \circ \varphi=p$.

Remark 2.5. More generally, the projection

$$
p: \mathcal{F}_{k+1}^{k, n} \longrightarrow \mathcal{F}_{k}^{k-1, n},\left(x_{1}, \ldots, x_{k+1}\right) \mapsto\left(x_{1}, \ldots, x_{k}\right)
$$

is a locally trivial fibration with simply connected fiber $\mathbb{C} P^{n} \backslash \mathbb{C} P^{k-1}$.

Proof. The proof is similar to the previous one: take $\left(x_{1}^{0}, \ldots, x_{k+1}^{0}\right) \in \mathcal{F}_{k}^{k, n}$ and fix $x_{k+2}^{0}, . ., x_{n+2}^{0}$ such that any $n+1$ points of the set $\left\{x_{1}^{0}, . ., x_{k+1}^{0}, . ., x_{n+2}^{0}\right\}$ are independent. Define the open neighborhood $\mathcal{U}$ of $\left(x_{1}^{0}, \ldots, x_{k}^{0}\right)$ by

$$
\mathcal{U}=\left\{\left(x_{1}, \ldots, x_{k}\right) \mid \text { the set }\left\{x_{1}, . ., x_{k}, x_{k+1}^{0}, . ., x_{n+2}^{0}\right\} \text { is in general position }\right\}
$$

and construct the trivialization as in Lemma 2.4. The fiber $\mathbb{C} P^{n} \backslash \mathbb{C} P^{k-1}$ is simply connected because the real codimension of $\mathbb{C} P^{k-1}$ is $\geq 4$ (for $k \leq n-1)$ and it is contractible for $k=n$.

Let $\mathcal{A}=\left(A_{1}, \ldots, A_{p}\right)$ be a sequence of subsets of $\{1, \ldots, k\}$ and the integers $d_{1}, \ldots, d_{p}$ given by $d_{j}=\left|A_{j}\right|-1, j=1, \ldots, p$. Let us define

$$
\mathcal{F}_{k}^{\mathcal{A}, n}=\left\{\left(x_{1}, \ldots, x_{k}\right) \in \mathcal{F}_{k}\left(\mathbb{C} P^{n}\right) \mid \operatorname{dim}<x_{i}>_{i \in A_{j}}=d_{j}\right\} .
$$

Example 2.6. (1) If $\mathcal{A}=\left\{A_{1}\right\}, A_{1}=\{1, \ldots, k\}$, then $\mathcal{F}_{k}^{\mathcal{A}, n}=\mathcal{F}_{k}^{k-1, n}$.

(2) if all $A_{i}$ have cardinality $\left|A_{i}\right| \leq 2$, then $\mathcal{F}_{k}^{\mathcal{A}, n}=\mathcal{F}_{k}\left(\mathbb{C} P^{n}\right)$.

(3) if $p \geq 2$ and $\left|A_{p}\right| \leq 2$, then $\mathcal{F}_{k}^{\left(A_{1}, \ldots, A_{p}\right), n}=\mathcal{F}_{k}^{\left(A_{1}, \ldots, A_{p-1}\right), n}$.

(4) if $p \geq 2$ and $A_{p} \subseteq A_{1}$, then $\mathcal{F}_{k}^{\left(A_{1}, \ldots, A_{p}\right), n}=\mathcal{F}_{k}^{\left(A_{1}, \ldots, A_{p-1}\right), n}$.

(5) $\mathcal{F}_{k}^{i, n}=\bigcup\left\{\mathcal{F}_{k}^{\mathcal{A}, n} \mid \mathcal{A}=\{A\}, A \in\left(\begin{array}{c}\{1, \ldots, k\} \\ i+1\end{array}\right)\right\}$.

(6) $Y_{k}^{n}=\bigcap\left\{\mathcal{F}_{k}^{\mathcal{A}, n} \mid \mathcal{A}=\{A\}, A \in\left(\begin{array}{c}\{1, \ldots, k\} \\ n+1\end{array}\right)\right\}$ is the space analyzed by Moulton in [M2].

Lemma 2.7. For $A=\{1, \ldots, i+1\}$ and $k>i+1$ the map

$$
P_{A}: \mathcal{F}_{k}^{\{A\}, i} \rightarrow \mathcal{F}_{i+1}^{i, i}, \quad\left(x_{1}, \ldots, x_{k}\right) \mapsto\left(x_{1}, \ldots, x_{i+1}\right)
$$

is a locally trivial fibration with fiber $\mathcal{F}_{k-i-1}\left(\mathbb{C} P^{i} \backslash\left\{x_{1}, \ldots, x_{i+1}\right\}\right)$. 
Proof. Choose $z_{i+2} \in \mathbb{C} P^{i}$ in general position with the set $\left(x_{1}, \ldots, x_{i+1}\right)$ and define the neighborhood $\mathcal{U}$ of $\left(x_{1}, \ldots, x_{i+1}\right) \in \mathcal{F}_{i+1}^{i, i}$ by $\mathcal{U}=\left\{\left(y_{1}, \ldots, y_{i+1}\right) \in\right.$ $\mathcal{F}_{i+1}^{i, i} \mid\left(y_{1}, \ldots, y_{i+1}, z_{i+2}\right)$ are in general position $\}$. There exists a unique projective isomorphism $F_{y}: \mathbb{C} P^{i} \rightarrow \mathbb{C} P^{i}$, which depends continuously on $y=\left(y_{1}, \ldots, y_{i+1}\right)$, such that

$$
F_{y}\left(x_{1}, \ldots, x_{i+1}, z_{i+2}\right)=\left(y_{1}, \ldots, y_{i+1}, z_{i+2}\right)
$$

and this gives a local trivialization

$$
\begin{aligned}
f: P_{A}^{-1}(\mathcal{U}) & \rightarrow \mathcal{U} \times \mathcal{F}_{k-i-1}\left(\mathbb{C} P^{i} \backslash\left\{x_{1}, \ldots, x_{i+1}\right\}\right) \\
\left(y_{1}, \ldots, y_{k}\right) & \mapsto\left(\left(y_{1}, \ldots, y_{i+1}\right), F_{y}^{-1}\left(y_{i+2}, \ldots, y_{k}\right)\right)
\end{aligned}
$$

which satisfies $p r_{1} \circ f=P_{A}$.

Remark 2.8. If $k=i+1$, then $P_{A}$ is the identity map.

Given $\mathcal{A}=\left(A_{1}, \ldots, A_{p}\right), d=\left(d_{1}, \ldots, d_{p}\right)$ as before and an index $h \in$ $\{1, \ldots, k\}$, we define $\mathcal{A}^{\prime}=\left(A_{1}^{\prime}, \ldots, A_{p}^{\prime}\right), d=\left(d_{1}^{\prime}, \ldots, d_{p}^{\prime}\right)$ by:

$$
A_{j}^{\prime}=\left\{\begin{array}{l}
A_{j}, \text { if } h \notin A_{j} \\
A_{j} \backslash\{h\}, \text { if } h \in A_{j}
\end{array}, \quad d_{j}^{\prime}=\left\{\begin{array}{l}
d_{j}, \text { if } h \notin A_{j} \\
d_{j}-1, \text { if } h \in A_{j}
\end{array} .\right.\right.
$$

Lemma 2.9. The map

$$
p_{h}: \mathcal{F}_{k}^{\mathcal{A}, n} \rightarrow \mathcal{F}_{k-1}^{\mathcal{A}^{\prime}, n},\left(x_{1}, \ldots, x_{k}\right) \mapsto\left(x_{1}, \ldots, \widehat{x_{h}}, \ldots, x_{k}\right)
$$

has local sections with path-connected fibers.

Proof. Let us suppose that $h=k$ and $k \in\left(A_{1} \cap \ldots \cap A_{l}\right) \backslash\left(A_{l+1} \cup \ldots \cup A_{p}\right)$. Then the fiber of the map $p r_{k}: \mathcal{F}_{k}^{\mathcal{A}, n} \rightarrow \mathcal{F}_{k-1}^{\mathcal{A}^{\prime}, n}$ is

$$
p r_{k}^{-1}\left(x_{1}, \ldots, x_{k-1}\right) \approx \mathbb{C} P^{n} \backslash\left(L_{1}^{\prime} \cup \ldots \cup L_{l}^{\prime} \cup\left\{x_{1}, \ldots, x_{k-1}\right\}\right)
$$

where $L_{j}^{\prime}=\operatorname{span}\left(x_{i}\right)_{i \in A_{j}^{\prime}}$. Even in the case when $\operatorname{dim} L_{j}=n$, we have $\operatorname{dim} L_{j}^{\prime}<n$, hence the fiber is path-connected and nonempty. Fix a base point $x=\left(x_{1}, \ldots, x_{k-1}\right) \in \mathcal{F}_{k-1}^{\mathcal{A}^{\prime}, n}$ and choose $x_{k} \in \mathbb{C} P^{n} \backslash\left(L_{1}^{\prime} \cup \ldots \cup L_{p}^{\prime} \cup\right.$ $\left.\left\{x_{1}, \ldots, x_{k-1}\right\}\right)$. There are neighborhoods $W_{j}$ of $L_{j}^{\prime}(j=1, \ldots, l)$ such that $x_{k} \notin L_{j}^{\prime \prime}$ if $L_{j}^{\prime \prime} \in W_{j}$; we take a constant local section

$$
\begin{gathered}
s: W=g^{-1}\left(\left(\mathbb{C} P^{n} \backslash\left\{x_{k}\right\}\right)^{k-1} \times \prod_{i=1}^{l} W_{i}\right) \rightarrow \mathcal{F}_{k}^{\mathcal{A}, n} \\
\left(y_{1}, \ldots, y_{k-1}\right) \mapsto\left(y_{1}, \ldots, y_{k-1}, x_{k}\right),
\end{gathered}
$$

where the continuous map $g$ is given by:

$$
\begin{gathered}
g: \mathcal{F}_{k-1}^{\mathcal{A}^{\prime}, n} \rightarrow\left(\mathbb{C} P^{n}\right)^{k-1} \times G r^{d_{1}^{\prime}}\left(\mathbb{C} P^{n}\right) \times \ldots \times G r^{d_{l}^{\prime}}\left(\mathbb{C} P^{n}\right) \\
\left(y_{1}, \ldots, y_{k-1}\right) \mapsto\left(y_{1}, \ldots, y_{k-1}, L_{1}^{\prime \prime}, \ldots, L_{p}^{\prime \prime}\right),
\end{gathered}
$$

and $L_{j}^{\prime \prime}=\operatorname{span}\left\{y_{i}\right\}_{i \in A_{j}^{\prime}}$ for $j=1, \ldots, l$. 
Lemma 2.10. Let $p: B \rightarrow C$ be a continuous map with local sections such that $C$ is path-connected and $p^{-1}(y)$ are path-connected for all $y \in C$. Therefore $B$ is path-connected.

Proof. For any $b \in B$ there exists a neighborhood $W$ of $p(b)$ and a section $s: W \rightarrow B$ such that $s(p(b))=b_{0}$. We can join $b$ with any point $b^{\prime}$ in $V=p^{-1}(W)$ by a continuous path: $b$ to $b_{0}$ and $b^{\prime}$ to $b_{0}^{\prime}=s\left(p\left(b^{\prime}\right)\right)$ in fibers and $b_{0}$ to $b_{0}^{\prime}$ using a path in $C$ and the section $s$. For any $b_{1}, b_{2} \in B$, a path in $C$ from $p\left(b_{1}\right)$ to $p\left(b_{2}\right)$ can be covered by finite open sets of the type described before and next we apply the previous construction.

Proposition 2.11. The space $\mathcal{F}_{k}^{\mathcal{A}, n}$ is path-connected.

Proof. Use the previous Lemma and induction on $p$ and $d_{1}+d_{2}+\ldots+d_{p}$. If $p=1$, use Lemma 2.7 and the space $\mathcal{F}_{i+1}^{i, i}$ which is path-connected. If $A_{p}$ is not included in $A_{1}$ and $d_{p} \geq 3$, delete a point in $A_{p} \backslash A_{1}$ and use Lemma 2.9 and 2.10, If $A_{p} \subset A_{1}$ or $d_{p} \leq 2$, use Example 2.6, (3) and (4).

Lemma 2.12. The homotopy class of the map

$$
\gamma: S^{1} \longrightarrow \mathcal{F}_{k+1}\left(\mathbb{C} P^{1}\right), \gamma(z)=([z: 1],[2 z: 1], \ldots,[k z: 1],[1: 0])
$$

corresponds to the following pure braid in $\pi_{1}\left(\mathcal{F}_{k+1}\left(\mathbb{C} P^{1}\right)\right)$ :

$$
[\gamma]=\alpha_{12}\left(\alpha_{13} \alpha_{23}\right) \ldots\left(\alpha_{1 k} \alpha_{2 k} \ldots \alpha_{k-1, k}\right)=D_{k}
$$

Proof. The loop $\gamma_{k+1}$ in $\mathcal{F}_{k+1}\left(\mathbb{C} P^{1}\right)$ given by

$$
z \longmapsto([z: 1],[2 z: 1], \ldots,[k z: 1],[1: 0])
$$

fixes the point at $\infty$ and gives the pure braid in $\mathbb{C}$

$$
t \longmapsto\left(e^{2 \pi i t}, 2 e^{2 \pi i t}, \ldots, k e^{2 \pi i t}\right)
$$

By induction we assume that the class $\left[\gamma_{k}\right]$ in $\mathcal{F}_{k}\left(\mathbb{C} P^{1}\right)$ is given by the element $\left(\sigma_{1} \ldots \sigma_{k-2}\right) \ldots\left(\sigma_{1} \sigma_{2}\right) \sigma_{1}^{2}\left(\sigma_{2} \sigma_{1}\right) \ldots\left(\sigma_{k-2} \ldots \sigma_{1}\right)$, where the $k$-points in $\mathbb{C} P^{1}$ are $2,3, \ldots, k$ and $\infty$. In $\mathcal{F}_{k+1}\left(\mathbb{C} P^{1}\right)$ we introduce a new strand corresponding to the point 1 . The image of the $j$-strand $t \rightarrow\left[j e^{2 \pi i t}: 1\right]$ lies on the cylinder $|z|=j, t \in[0,1]$, therefore the image of the strand corresponding to 1 is interior to all the other cylinders and can be deformed to a straight line segment, like in the next figure 


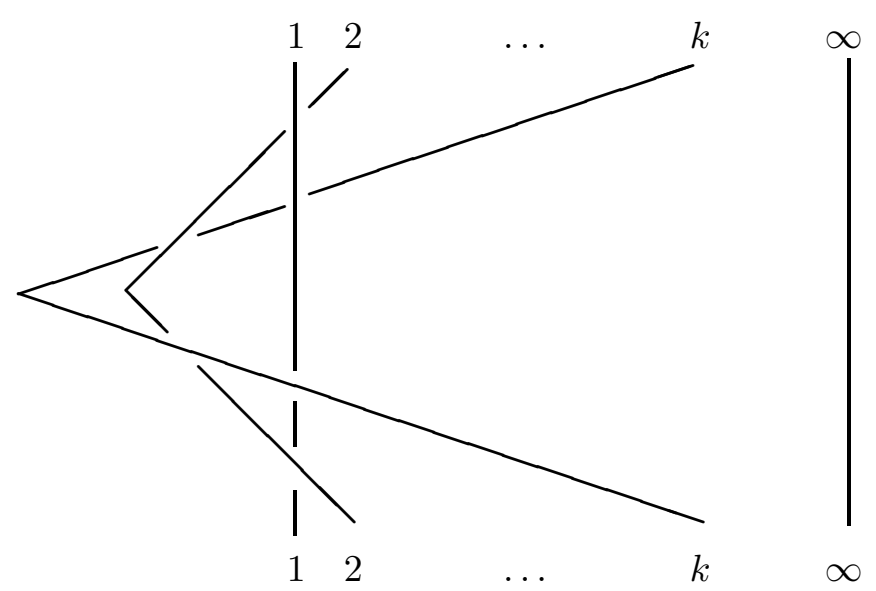

In the diagram of the pure braid $\left[\gamma_{k+1}\right]$ we can start with $k-1$ intersections of first strand with the second,..., with the $k$-th strand, next add the diagram of $\left[\gamma_{k}\right]$ (the first strand corresponding to 1 is in the $k$-th position, so the word $\left[\gamma_{k}\right]$ is unchanged) and end with the second intersections of the first strand with the other $k-1$ strands. This gives the representation of $\left[\gamma_{k+1}\right]=\left(\sigma_{1} \ldots \sigma_{k-1}\right)\left[\gamma_{k}\right]\left(\sigma_{k-1} \ldots \sigma_{1}\right)$. Hence

$$
\begin{aligned}
{\left[\gamma_{k+1}\right] } & =\left(\sigma_{1} \sigma_{2} . . \sigma_{k-1}\right) \ldots\left(\sigma_{1} \sigma_{2} \sigma_{3}\right)\left(\sigma_{1} \sigma_{2}\right) \sigma_{1}^{2}\left(\sigma_{2} \sigma_{1}\right)\left(\sigma_{3} \sigma_{2} \sigma_{1}\right) \ldots\left(\sigma_{k-1} . . \sigma_{2} \sigma_{1}\right) \\
& =\alpha_{12}\left(\alpha_{13} \alpha_{23}\right) \ldots\left(\alpha_{1 k} \alpha_{2 k} . . \alpha_{k-1, k}\right)=D_{k}=\Delta_{k}^{2}
\end{aligned}
$$

(see Appendix for the last equalities).

\section{Proofs of Theorem 1.1 and Theorem 1.3}

The complex Grassmannian manifolds $G r^{k}\left(\mathbb{C} P^{n}\right)$ are simply connected and the second homotopy group is stable $(n \geq k+1)$

$$
\mathbb{Z} \cong \pi_{2}\left(\mathbb{C} P^{1}\right) \stackrel{\cong}{\rightarrow} \pi_{2}\left(\mathbb{C} P^{k+1}\right) \stackrel{\cong}{\rightarrow} \pi_{2}\left(G r^{k}\left(\mathbb{C} P^{k+1}\right)\right) \stackrel{\cong}{\rightarrow} \pi_{2}\left(G r^{k}\left(\mathbb{C} P^{n}\right)\right),
$$

so we can choose the map

$$
\begin{gathered}
g:\left(D^{2}, S^{1}\right) \rightarrow\left(G r^{k}\left(\mathbb{C} P^{n}\right), L_{1}\right) \\
g(z)=L_{z}, \quad L_{z}:(1-|z|) X_{0}-z X_{1}=0, X_{k+2}=\ldots=X_{n}=0
\end{gathered}
$$

as a generator of $\pi_{2}\left(G r^{k}\left(\mathbb{C} P^{n}\right)\right)$.

Lemma 3.1. For $i \leq \min \{k, n-1\}$ we have

$$
\pi_{1}\left(\mathcal{F}_{k+1}^{i, i+1}\right) \cong \pi_{1}\left(\mathcal{F}_{k+1}^{i, n}\right) \text {. }
$$

Proof. The canonical embedding

$$
\mathbb{C} P^{i+1} \longrightarrow \mathbb{C} P^{n}, \quad\left[z_{0}: \ldots: z_{i}\right] \mapsto\left[z_{0}: \ldots: z_{i}: 0: \ldots: 0\right]
$$

induces the following commutative diagram of fibrations 


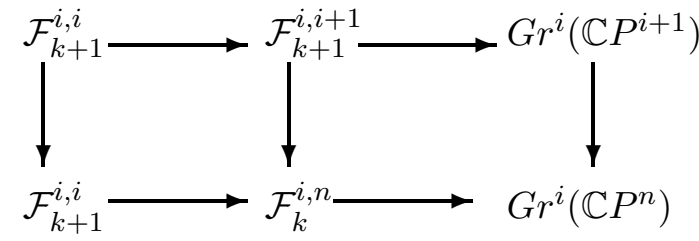

and the result is obtained from the commutative diagram of homotopy groups

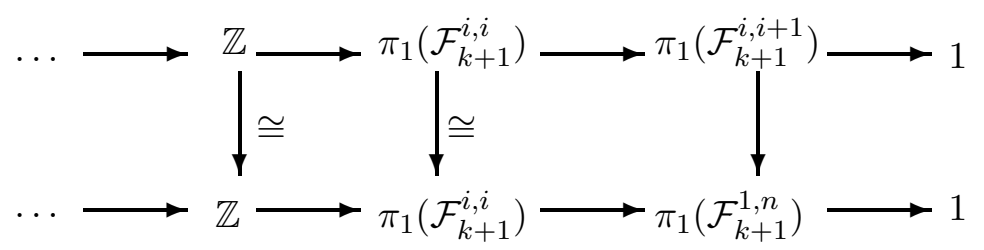

The top dimensional strata are simply connected.

Lemma 3.2. For any $k$ we have $\pi_{1}\left(\mathcal{F}_{k}^{k-1, k}\right) \cong \pi_{1}\left(\mathcal{F}_{k+1}^{k, k}\right)=1$.

Proof. The proof is by induction on $k$ and uses the fibration of Lemma 2.4 with contractible fiber $\mathbb{C} P^{k} \backslash \mathbb{C} P^{k-1}$ and the fibration

$$
\mathcal{F}_{k}^{k-1, k-1} \hookrightarrow \mathcal{F}_{k}^{k-1, k} \longrightarrow G r^{k-1}\left(\mathbb{C} P^{k}\right) .
$$

For $k=1, \pi_{1}\left(\mathcal{F}_{1}^{0,1}\right)=\pi_{1}\left(\mathbb{C} P^{1}\right)=1$ and $\pi_{1}\left(\mathcal{F}_{2}^{1,1}\right)=\pi_{1}\left(\mathcal{F}_{2}\left(\mathbb{C} P^{1}\right)\right)=1$. The later fibration gives $\pi_{1}\left(\mathcal{F}_{k}^{k-1, k}\right)=1$ and the former gives $\pi_{1}\left(\mathcal{F}_{k+1}^{k, k}\right)=1$.

The next result covers the simply connectedness cases of Theorem 1.1 .

Proposition 3.3. $\pi_{1}\left(\mathcal{F}_{k}^{i, n}\right)=1$ for $i \geq 2$ and $k \geq i+1$.

Proof. To prove that $\mathcal{F}_{k}^{i, i}$ is simply connected we use Seifert-Van Kampen theorem [H] for the finite open covering $\mathcal{F}_{k}^{i, i}=\bigcup\left\{\mathcal{F}_{k}^{\mathcal{A}, i} \mid \mathcal{A}=\{A\}, A \in\right.$ $\left.\left(\begin{array}{c}\{1, \ldots, k\} \\ i+1\end{array}\right)\right\}$. By Lemma 3.2 we have $\pi_{1}\left(\mathcal{F}_{i+1}^{i, i}\right)=1$ and using fibrations as in Lemma 2.7 we obtain

$$
\pi_{1}\left(\mathcal{F}_{k-i-1}\left(\mathbb{C} P^{i} \backslash\{i+1 \text { points }\}\right)\right) \rightarrow \pi_{1}\left(\mathcal{F}_{k}^{\{A\}, i}\right) \rightarrow \pi_{1}\left(\mathcal{F}_{i+1}^{i, i}\right),
$$

all the pieces of the covering are simply connected: the fiber $\mathcal{F}_{k-i-1}\left(\mathbb{C} P^{i} \backslash\right.$ $\{i+1$ points $\}$ ) is simply connected for $i \geq 2$ because $\mathbb{C} P^{i} \backslash\{i+1$ points $\}$ is simply connected (for the special case $k=i+1, \mathcal{F}_{k}^{\{A\}, i}=\mathcal{F}_{i+1}^{i, i}$ ). Also, every intersection $\mathcal{F}_{k}^{\left\{A_{1}\right\}, i} \cap \ldots \cap \mathcal{F}_{k}^{\left\{A_{r}\right\}, i}=\mathcal{F}_{k}^{\mathcal{A}, i}$ (where $\mathcal{A}=\left\{A_{1}, \ldots, A_{r}\right\}$ ) is path connected. Using the fibration in the proof of Lemma $3.1, \pi_{1}\left(\mathcal{F}_{k}^{i, i}\right)=1$ implies $\pi_{1}\left(\mathcal{F}_{k}^{i, n}\right)=1$ for $n \geq i \geq 2$. 
Using the geometrical fibrations introduce in previous section we start the inductive proof of Theorem 1.1.

3.1. Proof of Theorem 1.1. From Lemma 3.2 $\mathcal{F}_{k+1}^{k, k}, \mathcal{F}_{k+1}^{k, k+1}$ are simply connected spaces, and for $k \geq 1 \mathcal{F}_{k+1}^{1,1}=\mathcal{F}_{k+1}\left(\mathbb{C} P^{1}\right)$, so we have the classical result (see $[\mathrm{F}]$ )

$$
\pi_{1}\left(\mathcal{F}_{k+1}^{1,1}\right)=<\alpha_{i j}, 1 \leq i<j \leq k \mid(Y B 3),(Y B 4), D_{k}^{2}=1>.
$$

Using Proposition 3.3 we have $\pi_{1}\left(\mathcal{F}_{k}^{i, n}\right)=1$ for $n \geq i \geq 2, k \geq i+1$ and Lemma 3.1 implies $\pi_{1}\left(\mathcal{F}_{k+1}^{1,2}\right) \cong \pi_{1}\left(\mathcal{F}_{k+1}^{1, n}\right), \quad(k \geq 1)$, so we have to compute $\mathcal{F}_{k+1}^{1,2}$. Consider the following fibration (see Proposition 2.2)

$$
p: \mathcal{F}_{k+1}^{1,2} \rightarrow G r^{1}\left(\mathbb{C} P^{2}\right)
$$

with fiber $\mathcal{F}_{k+1}\left(\mathbb{C} P^{1}\right)$. Since $\pi_{1}\left(G r^{1}\left(\mathbb{C} P^{2}\right)\right)=1$ and $\pi_{2}\left(G r^{1}\left(\mathbb{C} P^{2}\right)\right)=$ $\pi_{2}\left(\mathbb{C} P^{2}\right) \cong \mathbb{Z}$, the homotopy exact sequence

$$
\ldots \longrightarrow \pi_{2}\left(\mathcal{F}_{k+1}^{1,2}\right) \longrightarrow \mathbb{Z} \stackrel{\delta_{*}}{\longrightarrow} \pi_{1}\left(\mathcal{F}_{k+1}\left(\mathbb{C} P^{1}\right)\right) \longrightarrow \pi_{1}\left(\mathcal{F}_{k+1}^{1,2}\right) \longrightarrow 1
$$

gives $\pi_{1}\left(\mathcal{F}_{k+1}^{1,2}\right)=\pi_{1}\left(\mathcal{F}_{k+1}\left(\mathbb{C} P^{1}\right)\right) /\left\langle\operatorname{Im} \delta_{*}\right\rangle$.

Let $[g]$ be the generator of $\pi_{2}\left(G r^{1}\left(\mathbb{C} P^{2}\right), L_{1}\right), L_{1}$ is the line $X_{1}=0$,

$$
g:\left(D^{2}, S^{1}\right) \longrightarrow\left(G r^{1}\left(\mathbb{C} P^{2}\right), L_{1}\right), z \longmapsto L_{z}:(1-|z|) X_{0}-z X_{1}=0 .
$$

We choose the lift $\widetilde{g}$

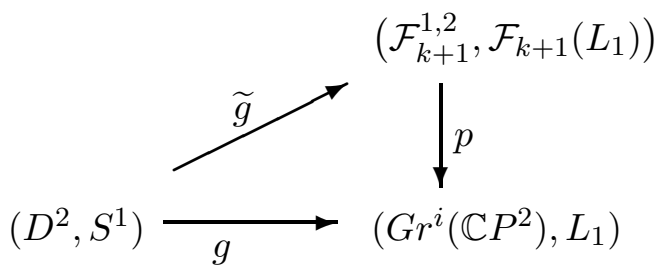

$\widetilde{g}(z)=\left([z: 1-|z|: 1],\left[z: 1-|z|: \frac{1}{2}\right], \ldots,\left[z: 1-|z|: \frac{1}{k}\right],[z, 1-|z|, 0]\right)$ and the image $\delta_{*}[g]$ is given by the restriction $\gamma=\left.\widetilde{g}\right|_{S^{1}}: S^{1} \longrightarrow \mathcal{F}_{k+1}\left(\mathbb{C} P^{1}\right)$

$\gamma(z)=\left([z: 1],\left[z: \frac{1}{2}\right], \ldots,\left[z: \frac{1}{k}\right],[1: 0]\right)=([z: 1],[2 z: 1], \ldots,[k z: 1],[1: 0])$.

By the Lemma 2.12, $[\gamma]=\alpha_{12} \alpha_{13} \alpha_{23} \ldots \alpha_{1 k} \ldots \alpha_{k-1, k}=D_{k}$, hence we obtain

$$
\begin{aligned}
\pi_{1}\left(\mathcal{F}_{k+1}^{1,2}\right) & =\pi_{1}\left(\mathcal{F}_{k+1}\left(\mathbb{C} P^{1}\right)\right) /\left\langle\operatorname{Im} \delta_{*}\right\rangle \\
& =\left\langle\alpha_{i j}, i \leq i<j \leq k \mid(Y B 3)_{k},(Y B 4)_{k}, D_{k}^{2}=1, D_{k}=1\right\rangle \\
& =\left\langle\alpha_{i j}, i \leq i<j \leq k \mid(Y B 3)_{k},(Y B 4)_{k}, D_{k}=1\right\rangle .
\end{aligned}
$$

Proof of Corollary $\mathbf{1 . 2}$. In $\pi_{1}\left(\mathcal{F}_{k+1}^{1,1}\right)_{a b}$ we have $\left(\begin{array}{l}k \\ 2\end{array}\right)$ generators $\left(a_{i j}\right)_{1 \leq i<j \leq k}$ and one relation: $2\left(a_{12}+a_{13}+a_{23}+\ldots+a_{k-1, k}\right)=0$. Changing the last generator $a_{k-1, k}$ with $a=a_{12}+a_{13}+a_{23}+\ldots+a_{k-1, k}$ we obtain a presentation of $\mathbb{Z}^{\left(\begin{array}{c}k \\ 2\end{array}\right)-1} \oplus \mathbb{Z}_{2}$. In the second case $a_{k-1, k}$ can be eliminated. 
Example 3.4. For $k=2$ we have $\pi_{1}\left(\mathcal{F}_{3}^{1,2}\right)=1, \pi_{2}\left(\mathcal{F}_{3}^{1,2}\right) \cong \mathbb{Z}$; for $k=3$, $\pi_{1}\left(\mathcal{F}_{4}^{1,2}\right) \cong \mathbb{F}_{2}$

For $k=2, \pi_{2}\left(\mathcal{F}_{3}\left(\mathbb{C} P^{1}\right)\right)=1$ (see [B2] $)$. Using $\pi_{2}\left(G r^{1}\left(\mathbb{C} P^{2}\right)\right) \cong \mathbb{Z}$ and the fibration $\mathcal{F}_{3}\left(\mathbb{C} P^{1}\right) \rightarrow \mathcal{F}_{3}^{1,2} \rightarrow G r^{1}\left(\mathbb{C} P^{2}\right)$, we have

$$
0 \longrightarrow \pi_{2}\left(\mathcal{F}_{3}^{1,2}\right) \longrightarrow \mathbb{Z} \stackrel{\delta_{*}}{\longrightarrow} \mathbb{Z}_{2} \longrightarrow \pi_{1}\left(\mathcal{F}_{3}^{1,2}\right) \longrightarrow 1 ;
$$

since the image of $\delta_{*}$ contains $D_{2}=\alpha_{12}$ (see Lemma 2.12), $\delta_{*}$ is surjective and $\pi_{1}\left(\mathcal{F}_{3}^{1,2}\right)=1$ and also $\pi_{2}\left(\mathcal{F}_{3}^{1,2}\right)=\mathbb{Z}$.

For $k=3, \pi_{1}\left(\mathcal{F}_{4}^{1,2}\right)$ is generated by $\alpha_{12}, \alpha_{13}, \alpha_{23}$ with defining relations

$$
\alpha_{12} \alpha_{13} \alpha_{23}=\alpha_{13} \alpha_{23} \alpha_{12}=\alpha_{23} \alpha_{12} \alpha_{13}=1,
$$

therefore any of $\alpha_{i j}$ can be discarded: $\pi_{1}\left(\mathcal{F}_{4}^{1,2}\right) \cong \mathbb{F}\left(\alpha_{12}, \alpha_{23}\right) \cong \mathbb{F}\left(\alpha_{13}, \alpha_{23}\right) \cong$ $\mathbb{F}\left(\alpha_{12}, \alpha_{13}\right)$.

Remark 3.5. For any continuous maps $f, g: S^{2} \rightarrow S^{2}$ without fixed points, there is an $x \in S^{2}$ such that $f(x)=g(x)$. More generally, for any map $F: S^{2} \rightarrow \mathcal{F}_{3}\left(S^{2}\right)$, the three projections $\mathrm{pr}_{j} \circ F$ are homotopically trivial.

Proof. The composition $\pi_{2}\left(S^{2}\right) \underset{F_{*}}{\underset{ }{\longrightarrow}} \pi_{2}\left(\mathcal{F}_{3}\left(S^{2}\right)\right) \underset{p r_{*}}{\longrightarrow} \pi_{2}\left(S^{2}\right)$ is trivial.

3.2. Proof of Theorem 1.3. First we have $\pi_{1}\left(\mathcal{C}_{k}^{i, n}\right) \cong \Sigma_{k}$ for $2 \leq i \leq$ $\min (k+1, n)$ as a consequence of Theorem 1.1 and the regular covering $p: \mathcal{F}_{k}^{i, n} \longrightarrow \mathcal{C}_{k}^{i, n}$. Next we have

$$
\pi_{1}\left(\mathcal{C}_{k+1}^{1,1}\right)=\pi_{1}\left(\mathcal{C}_{k+1}\left(\mathbb{C} P^{1}\right)\right) \cong \mathcal{B}_{k+1} /\left\langle\sigma_{1} \ldots \sigma_{k-1} \sigma_{k}^{2} \sigma_{k-1} \ldots \sigma_{1}=1\right\rangle .
$$

Finally we have to compute $\pi_{1}\left(\mathcal{C}_{k+1}^{1, n}\right)$ for $n, k \geq 2$. The following commutative diagram of the fibrations and coverings

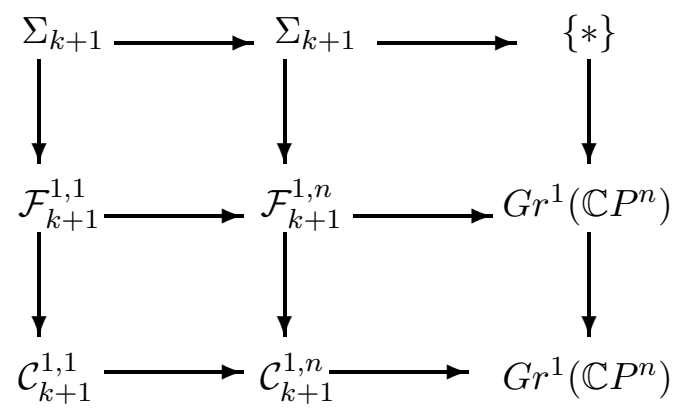

induces the commutative diagram of homotopy groups 


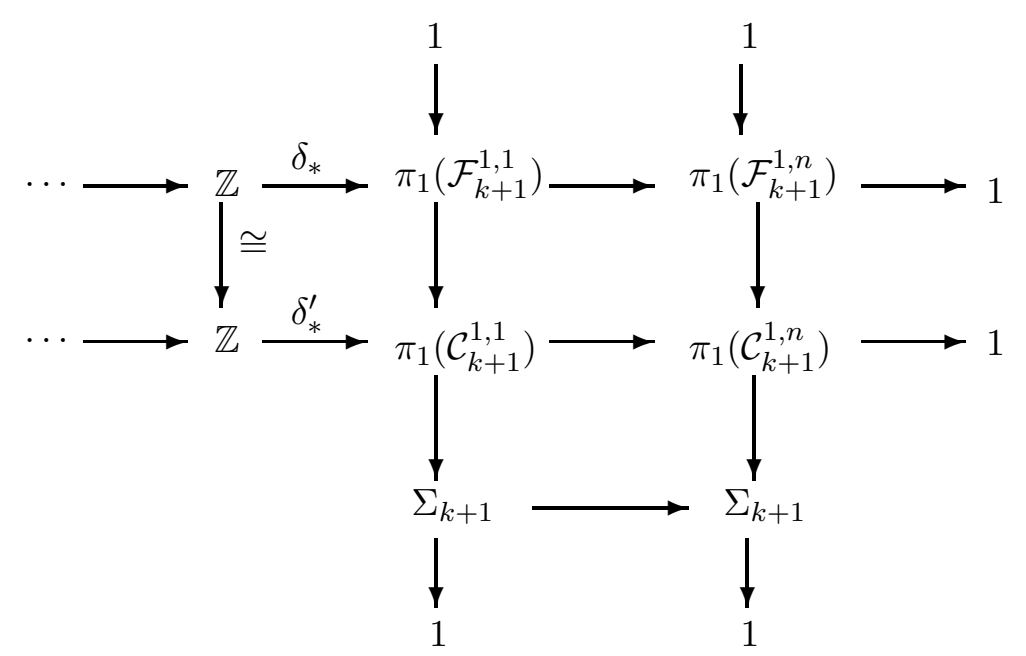

Since $\operatorname{Im} \delta_{*}=<D_{k}>$, the left square gives $\operatorname{Im} \delta_{*}^{\prime}=<\Delta_{k}^{2}>$, therefore $\pi_{1}\left(\mathcal{C}_{k+1}^{1, n}\right) \cong \mathcal{B}_{k+1} /\left\langle\sigma_{1} \ldots \sigma_{k-1} \sigma_{k}^{2} \sigma_{k-1} \ldots \sigma_{1},\left(\sigma_{1} \sigma_{2} \sigma_{1} \ldots \sigma_{k-1} \ldots \sigma_{1}\right)^{2}\right\rangle$.

Proof of Corollary 1.4. The abelianized group $\pi_{1}\left(\mathcal{C}_{k+1}^{1,1}\right)_{a b}$ is generated by $\left(s_{i}\right)_{i=1, \ldots, k}$ and the relations $s_{i+1}=s_{i}$ and $2\left(s_{1}+\ldots+s_{k}\right)=0$ and this gives a presentation of $\mathbb{Z}_{2 k}$. The group $\pi_{1}\left(\mathcal{C}_{k+1}^{1, n}\right)_{a b}$ has again one generator $s$ and the relations $2 k s=0$ and $k(k-1) s=0$. The greatest common divisor of $2 k$ and $k(k-1)$ is $k$ for $k$ even and $2 k$ for $k$ odd, hence the result.

\section{Pappus' Configurations}

Let us define the space of Pappus' configurations (in $\mathbb{C} P^{2}$ ) by $\mathcal{P}=\left\{\left(A_{1}, B_{1}, C_{1}, A_{2}, B_{2}, C_{2}\right) \in \mathcal{F}_{6}^{2,2} \mid\left(A_{i}, B_{i}, C_{i}\right) \in \mathcal{F}_{3}^{1,2}, A_{i}, B_{i}, C_{i} \neq I\right\}$ (here $I=d_{1} \cap d_{2}$, where $d_{i}$ is the line containing $A_{i}, B_{i}, C_{i}, i=1,2$ )

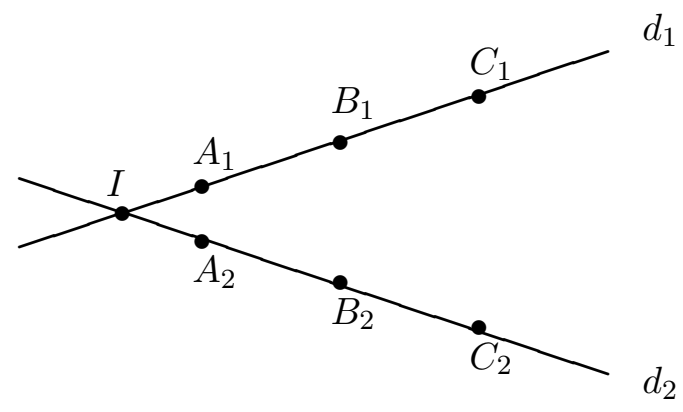


and also the space of Pappus' configurations with a fixed intersection point $I \in \mathbb{C} P^{2}$ as

$$
\mathcal{P}_{I}=\left\{\left(A_{1}, B_{1}, C_{1}, A_{2}, B_{2}, C_{2}\right) \in \mathcal{P} \mid d_{1} \cap d_{2}=I\right\} .
$$

In order to find the fundamental group of $\mathcal{P}$ and $\mathcal{P}_{I}$ we apply the same method: define two fibrations and use their homotopy exact sequences.

Lemma 4.1. The following projection

$$
\Gamma: \mathcal{P} \rightarrow \mathbb{C} P^{2},\left(A_{1}, B_{1}, C_{1}, A_{2}, B_{2}, C_{2}\right) \mapsto I=d_{1} \cap d_{2}
$$

is a locally trivial fibration with fiber $\mathcal{P}_{I}$.

Proof. Choose a line $l \subset \mathbb{C} P^{2} \backslash\left\{I^{0}\right\}$ and the neighborhood $\mathcal{U}_{l}=\mathbb{C} P^{2} \backslash l$ of $I^{0}$. For a point $I$ in this neighborhood and a Pappus' configuration $\left(A_{1}^{0}, B_{1}^{0}, C_{1}^{0}, A_{2}^{0}, B_{2}^{0}, C_{2}^{0}\right)$ on two lines $d_{1}^{0}, d_{2}^{0}$ containing $I^{0}$, construct lines $d_{1}, d_{2}$ and the configuration $\left(A_{1}, B_{1}, C_{1}, A_{2}, B_{2}, C_{2}\right)$ as follows: consider the points $D_{i}=l \cap d_{i}^{0}$ and $Q=l \cap I I^{0}$ and define $d_{i}=I D_{i}, A_{i}=d_{i} \cap Q A_{i}^{0}$ and in the same way $B_{i}, C_{i}(i=1,2)$. We describe this construction using coordinates to show that the map

$$
\left(A_{1}^{0}, B_{1}^{0}, C_{1}^{0}, A_{2}^{0}, B_{2}^{0}, C_{2}^{0}\right) \mapsto\left(A_{1}, B_{1}, C_{1}, A_{2}, B_{2}, C_{2}\right)
$$

has a continuous extension on the singular locus $\left(d_{1}^{0} \cup d_{2}^{0}\right) \backslash l$. Choose a projective frame such that $I^{0}=[0: 0: 1], l: X_{2}=0$. If $I=[s: t: 1]$ and $A_{i}^{0}=\left[n_{i}:-m_{i}: a_{i}\right], B_{i}^{0}=\left[n_{i}:-m_{i}: b_{i}\right], C_{i}^{0}=\left[n_{i}:-m_{i}: c_{i}\right]$ $\left(a_{i}, b_{i}, c_{i}\right.$ and 0 are distinct and also $\left.m_{1} n_{2} \neq m_{2} n_{1}\right)$, then we define $A_{i}=$ $\left[n_{i}+s a_{i}:-m_{i}+t a_{i}: a_{i}\right], B_{i}=\left[n_{i}+s b_{i}:-m_{i}+t b_{i}: b_{i}\right], C_{i}=\left[n_{i}+s c_{i}:\right.$ $\left.-m_{i}+t c_{i}: c_{i}\right](i=1,2)$, and these formulae agree with the geometrical construction given for nondegenerate positions of $I \in \mathbb{C} P^{2} \backslash\left(d_{1} \cup d_{2} \cup l\right)$. The trivialization over $\mathcal{U}_{l}$ is given by

$$
\begin{gathered}
\phi: \mathcal{U}_{l} \times \mathcal{P}_{I^{0}} \rightarrow \Gamma^{-1}\left(\mathcal{P}_{I^{0}}\right) \\
\phi\left(I,\left(A_{1}^{0}, B_{1}^{0}, C_{1}^{0}, A_{2}^{0}, B_{2}^{0}, C_{2}^{0}\right)\right)=\left(A_{1}, B_{1}, C_{1}, A_{2}, B_{2}, C_{2}\right) .
\end{gathered}
$$

Lemma 4.2. The projection

$$
\Lambda: \mathcal{P}_{I} \rightarrow \mathcal{F}_{2}\left(\mathbb{C} P^{1}\right),\left(A_{1}, B_{1}, C_{1}, A_{2}, B_{2}, C_{2}\right) \mapsto\left(d_{1}, d_{2}\right)
$$

is a locally trivial fibration with fiber $\mathcal{F}_{3}(\mathbb{C}) \times \mathcal{F}_{3}(\mathbb{C})$.

Proof. Choose a point $Q$ in $\mathbb{C} P^{2} \backslash\left(d_{1}^{0} \cup d_{2}^{0}\right)$ and the neighborhood $\mathcal{U}_{Q}=$ $\left\{\left(d_{1}, d_{2}\right) \in \mathcal{F}_{2}\left(\mathbb{C} P^{1}\right) \mid Q \notin d_{1} \cup d_{2}\right\}$. The trivialization over $\mathcal{U}_{Q}$ is given by

$$
\begin{gathered}
\psi: \mathcal{U}_{Q} \times \mathcal{F}_{3}\left(d_{1}^{0}\right) \times \mathcal{F}_{3}\left(d_{2}^{0}\right) \rightarrow \Lambda^{-1}\left(\mathcal{U}_{Q}\right) \\
\psi\left(\left(d_{1}, d_{2}\right),\left(A_{1}^{0}, B_{1}^{0}, C_{1}^{0}\right),\left(A_{2}^{0}, B_{2}^{0}, C_{2}^{0}\right)\right)=\left(A_{1}, B_{1}, C_{1}, A_{2}, B_{2}, C_{2}\right),
\end{gathered}
$$

where $A_{i}=d_{i} \cap Q A_{i}^{0}$ and similarly for $B_{i}, C_{i}(i=1,2)$. Obviously, $A_{i}, B_{i}, C_{i}$ and $I$ are four distinct points on $d_{i}$. 
Choose in $\mathcal{P}_{I=[0: 0: 1]}$ the base point $p_{0}=\left(A_{1}^{0}, B_{1}^{0}, C_{1}^{0}, A_{2}^{0}, B_{2}^{0}, C_{2}^{0}\right)$ where $A_{1}^{0}=[1: 0: 1], B_{1}^{0}=[2: 0: 1], C_{1}^{0}=[3: 0: 1], A_{2}^{0}=[0: 1: 1], B_{2}^{0}=[0: 2:$ 1], $C_{2}^{0}=[0: 3: 1]$.

Proposition 4.3. The fundamental group of $\mathcal{P}_{I}$ is given by

$$
\pi_{1}\left(\mathcal{P}_{I}, p_{0}\right) \cong \mathcal{P} \mathcal{B}_{3} \times \mathbb{F}_{2} .
$$

Proof. Lemma 4.2 gives the exact sequence

$$
\ldots \rightarrow \pi_{2}\left(\mathcal{F}_{2}\left(\mathbb{C} P^{1}\right)\right) \stackrel{\delta_{*}}{\longrightarrow} \pi_{1}\left(\mathcal{F}_{3}(\mathbb{C}) \times \mathcal{F}_{3}(\mathbb{C})\right) \longrightarrow \pi_{1}\left(\mathcal{P}_{\mathcal{I}}\right) \longrightarrow 1
$$

where the first group is cyclic generated by the homotopy class of the map

$$
\lambda:\left(D^{2}, S^{1}\right) \rightarrow \mathcal{F}_{2}\left(\mathbb{C} P^{1}\right), z \mapsto([1-|z|: z],[\bar{z}:|z|-1])
$$

(that means the two lines through $I=[0: 0: 1]$ are $d_{1}(z):(1-|z|) X_{0}+$ $z X_{1}=0$ and $\left.d_{2}(z): \bar{z} X_{0}+(|z|-1) X_{1}=0\right)$. The second group is generated by $\left\{\alpha_{i j}, \alpha_{i j}^{\prime}\right\}_{1 \leq i<j \leq 3}$ where
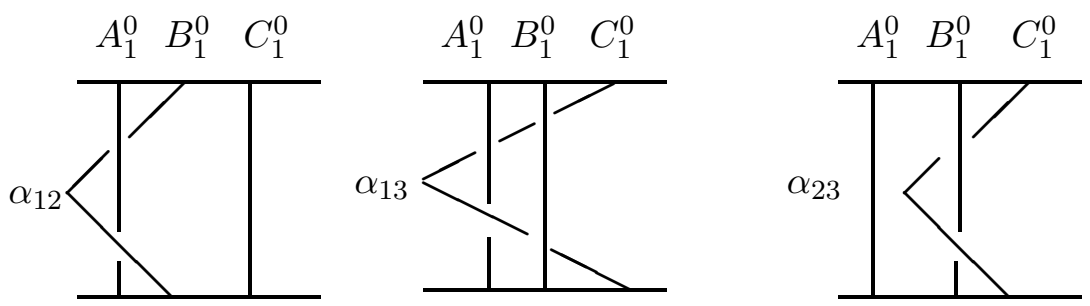

the points moving on the line $d_{1}^{0}: X_{1}=0$, respectively
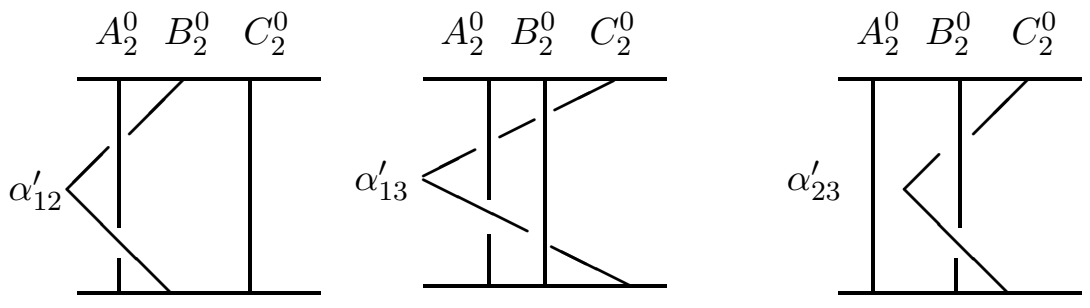

the points moving on the line $d_{2}^{0}: X_{0}=0$ and it has the presentation $\pi_{1}\left(\mathcal{F}_{3}(\mathbb{C}) \times \mathcal{F}_{3}(\mathbb{C})\right) \cong\left\langle\alpha_{i j}, \alpha_{i j}^{\prime}, 1 \leq i<j \leq 3 \mid(Y B 3)_{\alpha_{i j}},(Y B 3)_{\alpha_{i j}^{\prime}},\left[\alpha_{i j}, \alpha_{p q}^{\prime}\right]=1\right\rangle$.

We choose the lift $\tilde{\lambda}:\left(D^{2}, S^{1}\right) \rightarrow\left(\mathcal{P}_{I}, \mathcal{F}_{3}\left(d_{1}^{0} \backslash I^{0}\right) \times \mathcal{F}_{3}\left(d_{2}^{0} \backslash I^{0}\right)\right)$, $z \mapsto\left([z:|z|-1: 1],\left[z:|z|-1: \frac{1}{2}\right],\left[z:|z|-1: \frac{1}{3}\right],[1-|z|: \bar{z}: 1],[1-|z|:\right.$ $\left.\left.\bar{z}: \frac{1}{2}\right],\left[1-|z|: \bar{z}: \frac{1}{3}\right]\right)$, hence $\operatorname{Im} \delta_{*}$ is generated by the class of the map

$$
\left.\tilde{\lambda}\right|_{S^{1}}: S^{1} \rightarrow \mathcal{F}_{3}\left(d_{1}^{0} \backslash I^{0}\right) \times \mathcal{F}_{3}\left(d_{2}^{0} \backslash I^{0}\right), z \mapsto((z, 2 z, 3 z),(\bar{z}, 2 \bar{z}, 3 \bar{z})),
$$

and this is $\left(D_{3},\left(D_{3}^{\prime}\right)^{-1}\right)$ (see lemma 2.12). The group $\pi_{1}\left(\mathcal{P}_{I}, p_{0}\right)$ is generated by $\beta_{i j}, \beta_{p q}^{\prime}$, the images of $\alpha_{i j}, \alpha_{p q}^{\prime}$, with relations $(Y B 3)_{\beta_{i j}},(Y B 3)_{\beta_{i j}^{\prime}}$, 
$\left[\beta_{i j}, \beta_{p q}^{\prime}\right]=1$ and $\beta_{12}^{\prime} \beta_{13}^{\prime} \beta_{23}^{\prime}=\beta_{12} \beta_{13} \beta_{23}$. $\beta_{23}^{\prime}$ can be eliminated and we find the relations $(Y B 3)_{\beta_{i j}},\left[\beta_{i j}, \beta_{p q}^{\prime}\right]$, and this gives the presentation of the direct product $\mathcal{P B}_{3} \times \mathbb{F}_{2}$.

Remark 4.4. The generator $\beta_{12}$ of $\pi_{1}\left(\mathcal{P}_{I}\right)$ is given by the loop

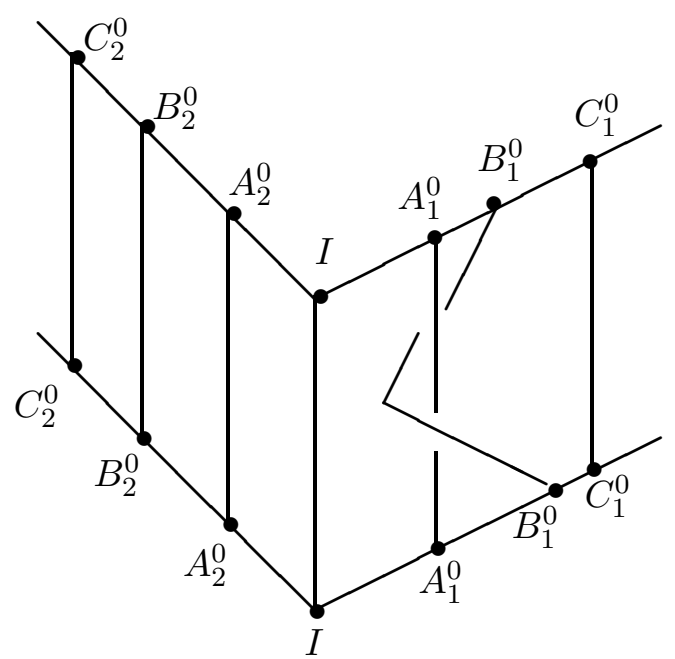

and there are similar pictures for $\beta_{13}, \beta_{23}, \beta_{12}^{\prime}, \beta_{13}^{\prime}$.

Proof of Theorem 1.5. Lemma 4.1 gives the exact sequence

$$
\ldots \longrightarrow \pi_{2}\left(\mathbb{C} P^{2}\right) \stackrel{\delta_{*}}{\longrightarrow} \pi_{1}\left(\mathcal{P}_{I}\right) \longrightarrow \pi_{1}(\mathcal{P}) \longrightarrow 1,
$$

where the first group is cyclic generated by the homotopy class of the map

$$
\gamma:\left(D^{2}, S^{1}\right) \rightarrow\left(\mathbb{C} P^{2},[0: 0: 1]\right), z \mapsto[0: 1-|z|: z] .
$$

We choose the lift $\widetilde{\gamma}:\left(D^{2}, S^{1}\right) \rightarrow\left(\mathcal{P}, \mathcal{P}_{I}\right), \widetilde{\gamma}(z)=([1: 0: 1],[2:|z|-1:$ $2-z],[3: 2|z|-2: 3-2 z],[0: 1-|z|+\bar{z}:|z|-1+z],[0: 1-|z|+2 \bar{z}:$ $2|z|-2+z],[0: 1-|z|+3 \bar{z}: 3|z|-3+z])$, hence $\operatorname{Im} \delta_{*}$ is generated by the class of the map $\left.\widetilde{\gamma}\right|_{S^{1}}: S^{1} \rightarrow \mathcal{P}_{I}, z \mapsto\left(\left[\begin{array}{lll}1 & : & :\end{array}\right],\left[\frac{2}{2-z}: 0:\right.\right.$ $\left.1],\left[\frac{3}{3-2 z}: 0: 1\right],\left[0: \bar{z}^{2}: 1\right],\left[0: 2 \bar{z}^{2}: 1\right],\left[0: 3 \bar{z}^{2}: 1\right]\right)$; the images of the loops $z \mapsto \frac{2}{2-z}, z \mapsto \frac{3}{3-z}$ are two cirles around the constant loop $z \mapsto 1$ and the first circle is included in the second one. As in Lemma 2.12 we find $\delta_{*}[\widetilde{\gamma}]=\left(\beta_{12} \beta_{13} \beta_{23}\right)\left(\beta_{12}^{\prime} \beta_{13}^{\prime} \beta_{23}^{\prime}\right)^{-2}=\left(\beta_{12} \beta_{13} \beta_{23}\right)^{-1}$, therefore $\beta_{23}$ can be eliminated and this gives the presentation of the direct product $\mathbb{F}_{2} \times \mathbb{F}_{2}$ (the generators are $\beta_{12}, \beta_{13}$ and $\beta_{12}^{\prime}, \beta_{13}^{\prime}$ ).

Define the space of Pappus' configurations (in $\mathbb{C} P^{n}$ ) by $\mathcal{P}^{(n)}=\left\{\left(A_{1}, B_{1}, C_{1}, A_{2}, B_{2}, C_{2}\right) \in \mathcal{F}_{6}^{2, n} \mid\left(A_{i}, B_{i}, C_{i}\right) \in \mathcal{F}_{3}^{1, n}, A_{i}, B_{i}, C_{i} \neq I\right\}$ (here $I=d_{1} \cap d_{2}$, where $d_{i}$ is the line containing $A_{i}, B_{i}, C_{i}, i=1,2$ ).

Theorem 4.5. $\pi_{1}\left(\mathcal{P}^{(n)}\right) \cong \pi_{1}(\mathcal{P}) \cong \mathbb{F}_{2} \times \mathbb{F}_{2}$. 
Proof. Using the fibration (the proof is like in Proposition 2.2)

$$
\mathcal{P}=\mathcal{P}^{(2)} \longrightarrow \mathcal{P}^{(n)} \longrightarrow G r^{2}\left(\mathbb{C} P^{n}\right)
$$

first we find that $\pi_{1}\left(\mathcal{P}^{(3)}\right) \cong \pi_{1}\left(\mathcal{P}^{(n)}\right)$ for $n \geq 3$ (like in Lemma 3.1) and secondly, in the fibration $\mathcal{P} \longrightarrow \mathcal{P}^{3} \longrightarrow G r^{2}\left(\mathbb{C} P^{3}\right)$, the boundary morphism $\pi_{2}\left(G r^{2}\left(\mathbb{C} P^{3}\right)\right) \rightarrow \pi_{1}(\mathcal{P})$ is trivial: take as generator of $\pi_{2}\left(G r^{2}\left(\mathbb{C} P^{2}\right)\right)$ the class of $\mu:\left(D^{2}, S^{1}\right) \rightarrow\left(G r^{2}\left(\mathbb{C} P^{3}\right), H_{1}\right), z \mapsto H_{z}:(1-|z|) X_{0}+z X_{3}=0$, and choose the lift $\widetilde{\mu}:\left(D^{2}, S^{1}\right) \rightarrow\left(\mathcal{P}^{(3)}, \mathcal{P}\right), z \mapsto([z: 0: 1:|z|-1],[2 z: 0:$ $1: 2|z|-2],[3 z: 0: 1: 3|z|-3],[0: 1: 1: 0],[0: 2: 1: 0],[0: 3: 1: 0])$, and $\delta_{*}\left[\left.\widetilde{\mu}\right|_{S^{1}}\right]=\beta_{12} \beta_{13} \beta_{23}=1$.

\section{APPENDIX}

In this Appendix we recollect different formulae in braid groups involving the braids $\sigma_{1} \ldots \sigma_{k}, \sigma_{k} \ldots \sigma_{1}$ (consecutive factors) and Garside braid $\Delta_{n}$ and also direct proofs of these relations scattered in the literature ([B2,G,M1]).

Lemma 5.1. (1) $\left(\sigma_{1} \ldots \sigma_{k}\right) \sigma_{i}=\sigma_{i+1}\left(\sigma_{1} \ldots \sigma_{k}\right)$ for $i=1, \ldots, k-1$;

(2) $\left(\sigma_{k} \ldots \sigma_{1}\right) \sigma_{i}=\sigma_{i-1}\left(\sigma_{k} \ldots \sigma_{1}\right)$ for $i=2, \ldots, k$;

(3) $\left(\sigma_{k} \ldots \sigma_{1} \sigma_{1} \ldots \sigma_{k}\right) \sigma_{j}=\sigma_{j}\left(\sigma_{k} \ldots \sigma_{1} \sigma_{1} \ldots \sigma_{k}\right)$, for $j=1, \ldots, k-1$.

Proof. These equalities are direct consequences of braid relations

$$
\begin{gathered}
\sigma_{i} \sigma_{j}=\sigma_{j} \sigma_{i}, \quad \forall i, j=1, \ldots, n-1 \text { with }|i-j| \geq 2 \\
\sigma_{i} \sigma_{i+1} \sigma_{i}=\sigma_{i+1} \sigma_{i} \sigma_{i+1} \text { for } i=1, \ldots, n-2 .
\end{gathered}
$$

In the next Lemma there are given some symmetric words in the diagram of Garside braid $\Delta_{n}$; the first one is the smallest in the length-lexicographic order, the last one is the greatest.

Lemma 5.2. We have the following representations of the fundamental braid $\Delta_{n}$

$$
\begin{aligned}
\Delta_{n} & =\sigma_{1}\left(\sigma_{2} \sigma_{1}\right) \ldots\left(\sigma_{n-1} \ldots \sigma_{1}\right) \\
& =\left(\sigma_{1} \ldots \sigma_{n-1}\right) \ldots\left(\sigma_{1} \sigma_{2}\right) \sigma_{1} \\
& =\left(\sigma_{n-1} \sigma_{n-2} \ldots \sigma_{1}\right)\left(\sigma_{n-1} \sigma_{n-2} \ldots \sigma_{2}\right) \ldots\left(\sigma_{n-1} \sigma_{n-2}\right) \sigma_{n-1} \\
& =\sigma_{n-1}\left(\sigma_{n-2} \sigma_{n-1}\right)\left(\sigma_{n-3} \sigma_{n-2} \sigma_{n-1}\right) \ldots\left(\sigma_{1} \ldots \sigma_{n-1}\right) .
\end{aligned}
$$

Proof. Let us denote $\Delta_{n}^{\prime}, \Delta_{n}^{\prime \prime}$ and $\Delta_{n}^{\prime \prime \prime}$ the second, the third and the fourth word respectively. We will prove by induction $\Delta_{n}^{\prime}=\Delta_{n}^{\prime \prime \prime}, \Delta_{n}^{\prime \prime}=\Delta_{n}, \Delta_{n}^{\prime \prime \prime}=$ $\Delta_{n}$. We will denote the shift of a word $\omega$ by $\Sigma \omega$ (for example, if $\omega=$ $\left.x_{1} x_{2} x_{4} x_{3}, \Sigma \omega=x_{2} x_{3} x_{5} x_{4}\right)$.

For $n=3, \Delta_{3}=\Delta_{3}^{\prime}=\sigma_{1} \sigma_{2} \sigma_{1}=\sigma_{2} \sigma_{1} \sigma_{2}=\Delta_{3}^{\prime \prime \prime}=\Delta_{3}^{\prime \prime}$. Suppose that for $k<n$, we have $\Delta_{k}=\Delta_{k}^{\prime}=\Delta_{k}^{\prime \prime}=\Delta_{k}^{\prime \prime \prime}$. Applying Lemma (5.1,(a)) on $\left(\sigma_{1} \ldots \sigma_{n-n}\right) \ldots\left(\sigma_{1} \sigma_{2}\right) \sigma_{1}$ we get 


$$
\begin{aligned}
\Delta_{n}^{\prime} & =\left(\sigma_{1} \ldots \sigma_{n-1}\right) \ldots\left(\sigma_{1} \sigma_{2}\right) \sigma_{1} \\
& =\left(\sigma_{2} \ldots \sigma_{n-1}\right) \ldots\left(\sigma_{2} \sigma_{3}\right) \sigma_{2}\left(\sigma_{1} \ldots \sigma_{n-1}\right) \\
& =\left(\Sigma \Delta_{n-1}^{\prime}\right)\left(\sigma_{1} \ldots \sigma_{n-1}\right) \\
& =\left(\Sigma \Delta_{n-1}^{\prime \prime \prime}\right)\left(\sigma_{1} \ldots \sigma_{n-1}\right)=\Delta_{n}^{\prime \prime \prime} .
\end{aligned}
$$

Applying Lemma (5.1, (b)) on $\left(\sigma_{n-1} \sigma_{n-2} \ldots \sigma_{2}\right) \ldots\left(\sigma_{n-1} \sigma_{n-2}\right) \sigma_{n-1}$ we get

$$
\begin{aligned}
\Delta_{n}^{\prime \prime} & =\left(\sigma_{n-1} \sigma_{n-2} \ldots \sigma_{1}\right)\left(\sigma_{n-1} \sigma_{n-2} \ldots \sigma_{2}\right) \ldots\left(\sigma_{n-1} \sigma_{n-2}\right) \sigma_{n-1} \\
& =\left(\sigma_{n-2} \ldots \sigma_{1}\right) \ldots\left(\sigma_{n-2} \sigma_{n-3}\right) \sigma_{n-2}\left(\sigma_{n-1} \ldots \sigma_{1}\right) \\
& =\Delta_{n-1}^{\prime \prime}\left(\sigma_{n-1} \ldots \sigma_{1}\right)=\Delta_{n-1}\left(\sigma_{n-1} \ldots \sigma_{1}\right)=\Delta_{n} .
\end{aligned}
$$

Finally, use the symmetry given by conjugation with $\Delta_{n}\left(\Delta_{n} \sigma_{i}=\sigma_{n-i} \Delta_{n}\right)$ :

$$
\Delta_{n}=\Delta_{n} \sigma_{1}\left(\sigma_{1} \sigma_{2}\right) \ldots\left(\sigma_{n-1} \ldots \sigma_{1}\right) \Delta_{n}^{-1}=\Delta_{n}^{\prime \prime \prime} .
$$

Now we give some formulae for $\Delta_{n}^{2}$ using braid generators $\sigma_{i}$ (the first one gives the smallest word ) and also pure braid generators $\alpha_{i j}$ (the last one is the smallest in the length lexicographic order given by $\alpha_{12}<\alpha_{13}<$ $\left.\ldots<\alpha_{n-1, n}\right)$.

Lemma 5.3. We have the following representations of $\Delta_{n}^{2}$

$$
\begin{aligned}
\Delta_{n}^{2} & =\sigma_{1}^{2}\left(\sigma_{2} \sigma_{1}^{2} \sigma_{2}\right) \ldots\left(\sigma_{n-1} \sigma_{n-2} \ldots \sigma_{2} \sigma_{1}^{2} \sigma_{2} \ldots \sigma_{n-2} \sigma_{n-1}\right) \\
& =\left(\sigma_{n-1} \ldots \sigma_{2} \sigma_{1}^{2} \sigma_{2} \ldots \sigma_{n-1}\right) \ldots\left(\sigma_{3} \sigma_{2} \sigma_{1}^{2} \sigma_{2} \sigma_{3}\right)\left(\sigma_{2} \sigma_{1}^{2} \sigma_{2}\right) \sigma_{1}^{2} \\
& =\left(\sigma_{1} \sigma_{2} \ldots \sigma_{n-1}\right) \ldots\left(\sigma_{1} \sigma_{2} \sigma_{3}\right)\left(\sigma_{1} \sigma_{2}\right) \sigma_{1}^{2}\left(\sigma_{2} \sigma_{1}\right)\left(\sigma_{3} \sigma_{2} \sigma_{1}\right) \ldots\left(\sigma_{n-1} \ldots \sigma_{2} \sigma_{1}\right) \\
& =\left(\sigma_{1} \ldots \sigma_{n-1}\right)^{n}=\left(\sigma_{n-1} \ldots \sigma_{1}\right)^{n} \\
& =\left(\alpha_{1 n} \alpha_{2 n} \ldots \alpha_{n-1, n}\right)\left(\alpha_{1 n-1} \ldots \alpha_{n-2, n-1}\right) \ldots\left(\alpha_{13} \alpha_{23}\right) \alpha_{12} \\
& =\alpha_{12}\left(\alpha_{13} \alpha_{23}\right) \ldots\left(\alpha_{1 n} \alpha_{2 n} \ldots \alpha_{n-1, n}\right) .
\end{aligned}
$$

Proof. Let $A_{n}, B_{n}, C_{n}, D_{n}, D_{n}^{\prime}, E_{n}, F_{n}$ be the above seven words respectively.

$$
\begin{aligned}
A_{n} & =\sigma_{1}^{2}\left(\sigma_{2} \sigma_{1}^{2} \sigma_{2}\right) \ldots\left(\sigma_{n-1} \sigma_{n-2} \ldots \sigma_{2} \sigma_{1}^{2} \sigma_{2} \ldots \sigma_{n-2} \sigma_{n-1}\right) \\
& =\Delta_{n-1}^{2}\left(\sigma_{n-1} \ldots \sigma_{2} \sigma_{1}\right)\left(\sigma_{1} \ldots \sigma_{n-1}\right) \\
& =\Delta_{n-1}\left(\Delta_{n}\right)\left(\sigma_{1} \ldots \sigma_{n-1}\right)=\Delta_{n-1}\left(\sigma_{n-1} \ldots \sigma_{1}\right) \Delta_{n}=\Delta_{n}^{2} \\
B_{n} & =\left(\sigma_{n-1} \ldots \sigma_{2} \sigma_{1}^{2} \sigma_{2} \ldots \sigma_{n-1}\right) \ldots\left(\sigma_{3} \sigma_{2} \sigma_{1}^{2} \sigma_{2} \sigma_{3}\right)\left(\sigma_{2} \sigma_{1}^{2} \sigma_{2}\right) \sigma_{1}^{2} \\
& =\left(\sigma_{n-1} \ldots \sigma_{2} \sigma_{1}\right)\left(\sigma_{1} \ldots \sigma_{n-1}\right) \Delta_{n-1}^{2} \\
& =\left(\sigma_{n-1} \ldots \sigma_{2} \sigma_{1}\right)\left(\sigma_{1} \ldots \sigma_{n-1}\right) \Delta_{n-1}^{\prime} \Delta_{n-1}^{\prime} \\
& =\left(\sigma_{n-1} \ldots \sigma_{2} \sigma_{1}\right) \Delta_{n}^{\prime} \Delta_{n-1}^{\prime}=\Delta_{n}\left(\sigma_{1} \ldots \sigma_{n-1}\right) \Delta_{n-1}^{\prime}=\Delta_{n}^{2}
\end{aligned}
$$

and

$$
C_{n}=\Delta_{n}^{\prime} \cdot \Delta_{n}=\Delta_{n}^{2}
$$

In the product of $n$ brackets of $D_{n}$ we move $\sigma_{n-1}$ from the first bracket $n-2$ times (just before the last bracket) and this becomes $\sigma_{1}$, then we continue 
this process with $\sigma_{n-1}$ of the second bracket and so on; we obtain

$$
\begin{aligned}
D_{n} & =\left(\sigma_{1} \sigma_{2} \ldots \sigma_{n-1}\right)^{n} \\
& =\left(\sigma_{1} \ldots \sigma_{n-2}\right)\left(\sigma_{1} \ldots \sigma_{n-1}\right) \ldots\left(\sigma_{1} \ldots \sigma_{n-1}\right) \sigma_{1}\left(\sigma_{1} \ldots \sigma_{n-1}\right) \\
& =\left(\sigma_{1} \ldots \sigma_{n-2}\right)\left(\sigma_{1} \ldots \sigma_{n-2}\right) \ldots\left(\sigma_{1} \ldots \sigma_{n-1}\right) \sigma_{2} \sigma_{1}\left(\sigma_{1} \ldots \sigma_{n-1}\right)=\ldots \\
& =\left(\sigma_{1} \ldots \sigma_{n-2}\right)\left(\sigma_{1} \ldots \sigma_{n-2}\right) \ldots\left(\sigma_{1} \ldots \sigma_{n-2}\right)\left(\sigma_{n-1} \ldots \sigma_{1}\right)\left(\sigma_{1} \ldots \sigma_{n-2}\right) \\
& =D_{n-1}\left(\sigma_{n-1} \ldots \sigma_{1}\right)\left(\sigma_{1} \ldots \sigma_{n-2}\right) \\
& =A_{n-1}\left(\sigma_{n-1} \ldots \sigma_{1}\right)\left(\sigma_{1} \ldots \sigma_{n-2}\right)=A_{n} . \\
D_{n}^{\prime}= & \Delta_{n} D_{n} \Delta_{n}^{-1}=D_{n} .
\end{aligned}
$$

Using $\alpha_{i j}=\sigma_{j-1} \sigma_{j-2} \ldots \sigma_{i+1} \sigma_{i}^{2} \sigma_{i+1}^{-1} \ldots \sigma_{j-1}^{-1}$, we obtain after cancelation

$$
\alpha_{1 n} \alpha_{2 n} \ldots \alpha_{n-1, n}=\left(\sigma_{n-1} \sigma_{n-2} \ldots \sigma_{1}\right)\left(\sigma_{1} \sigma_{2} \ldots \sigma_{n-1}\right)
$$

and

$$
\begin{aligned}
E_{n} & =\left(\alpha_{1 n} \alpha_{2 n} \ldots \alpha_{n-1, n}\right)\left(\alpha_{1 n-1} \ldots \alpha_{n-2, n-1}\right) \ldots\left(\alpha_{13} \alpha_{23}\right) \alpha_{12} \\
& =\left(\sigma_{n-1} \sigma_{n-2} . . \sigma_{1}\right)\left(\sigma_{1} . . \sigma_{n-1}\right)\left(\sigma_{n-2} . . \sigma_{1}\right)\left(\sigma_{1} . . \sigma_{n-2}\right) \ldots\left(\sigma_{2} \sigma_{1}\right)\left(\sigma_{1} \sigma_{2}\right) \sigma_{1}^{2} \\
& =B_{n},
\end{aligned}
$$

and

$F_{n}=F_{n-1}\left(\alpha_{1 n} \ldots \alpha_{n-1, n}\right)=B_{n-1}\left(\sigma_{n-1} \sigma_{n-2} \ldots \sigma_{1}\right)\left(\sigma_{1} \sigma_{2} \ldots \sigma_{n-1}\right)=B_{n}$.

\section{REFERENCES}

[A] Artin, E. (1947), Theory of braids, Ann. of Math. (2)48, pp. 101-126.

[B1] Berger, M. (1987), Geometry I, Springer-Verlarg Berlin.

[B2] Birman, Joan S. (1974), Braids, Links, and Mapping Class Groups, Annals of Mathematics vol. 82, Princeton University Press.

[F] Fadell, E.R, Husseini, S.Y. (2001), Geometry and Topology of Configuration Spaces, Springer Monographs in Mathematics, Springer-Verlarg Berlin.

[G] Garside, F.A. (1969), The braid groups and other groups, Quat. J. of Math. Oxford, $2^{e}$ ser. 20, 235-254.

[H] Hatcher, A. (2002), Algebraic Topology, Cambridge University Press.

[M1] Moran, S. (1983), The Mathematical Theory of Knots and Braids, North Holland Mathematics Studies, Vol 82 (Elsevier, Amsterdam).

[M2] Moulton, V. L. (1998), Vector Braids, J. Pure Appl. Algebra, 131, no. 3, 245-296.

${ }^{1}$ Abdus Salam School of Mathematical Sciences, GC University, LahorePakistan, and Institute of Mathematics Simion Stoilow, Bucharest-Romania, (Permanent address).

E-mail address: Barbu.Berceanu@imar.ro

${ }^{2}$ Abdus Salam School of Mathematical Sciences, GC University, LahorePAKISTAN.

E-mail address: saimashaa@gmail.com 